TRAVAIL

ET EMPLOI

\section{Travail et Emploi}

135 | juillet-septembre 2013

Expérimentations de terrain et politiques publiques du travail et de l'emploi. Apports récents et mises en perspective

\title{
Florence Jany-Catrice, La performance totale : nouvel esprit du capitalisme?
}

Villeneuve d'Ascq, Presses universitaires du Septentrion, coll. «Capitalismes-éthique-institutions », 2012

\section{Thierry Kirat}

\section{(2) OpenEdition}

\section{Journals}

Édition électronique

URL : http://journals.openedition.org/travailemploi/6098

DOI : $10.4000 /$ travailemploi.6098

ISSN : 1775-416X

Éditeur

DARES - Ministère du Travail

Édition imprimée

Date de publication : 15 septembre 2013

Pagination : 81-82

ISSN : 0224-4365

Référence électronique

Thierry Kirat, «Florence Jany-Catrice, La performance totale : nouvel esprit du capitalisme? », Travail et Emploi [En ligne], 135 I juillet-septembre 2013, mis en ligne le 01 juillet 2013, consulté le 22 septembre 2020. URL : http://journals.openedition.org/travailemploi/6098; DOI : https://doi.org/10.4000/ travailemploi.6098

Ce document a été généré automatiquement le 22 septembre 2020.

(c) Direction de l'animation de la recherche, des études et des statistiques (Dares) 


\section{Florence Jany-Catrice, $\mathrm{La}$ performance totale : nouvel esprit du capitalisme?}

Villeneuve d'Ascq, Presses universitaires du Septentrion, coll. «Capitalismes-éthique-institutions », 2012

\section{Thierry Kirat}

\section{RÉFÉRENCE}

Florence Jany-Catrice, La performance totale : nouvel esprit du capitalisme?, Villeneuve d'Ascq, Presses universitaires du Septentrion, coll. « Capitalismes-éthiqueinstitutions ", 2012, $176 \mathrm{p}$.

1 Les travaux de Florence Jany-Catrice sur les indicateurs de richesse menés avec Jean Gadrey ou Dominique Méda sont bien connus. Or, l'auteure est également spécialiste des services, notamment des services à la personne. Dans cet ouvrage, elle articule et donne de la cohérence à ses réflexions sur ces deux grandes questions : la mesure via des indicateurs et l'évaluation des services, plus particulièrement de ceux qui ont une dimension de care.

2 Tout au long de cet ouvrage relativement court (157 pages de texte), Florence JanyCatrice passe en revue de façon attentive une série de domaines dans lesquels la mesure de la performance par le biais d'indicateurs chiffrés est devenue une pratique courante ; qu'il s'agisse des services, des relations de travail, de la gestion publique, elle démontre et illustre qu'ils révèlent la domination de l'idée selon laquelle «la performance d'un individu, d'un système, d'un territoire, d'un pays, ne peut être envisagée autrement que par une transformation en données chiffrées» (p. 24). S'inscrivant dans la lignée de la sociologie de la quantification d'Alain Desrosières et d'Ève Chiapello, elle la met en relation avec une économie politique de la mesure (dans la lignée de Jean Gadrey), mais aussi avec l'économie des conventions (François 
Eymard-Duvernay, Laurent Thévenot); à l'encontre de la conception qui fait de la mesure l'image fidèle d'une réalité objective (qualifiée de "réaliste »), Florence JanyCatrice adopte une posture constructiviste, qui voit dans la mesure une construction, dont les conditions sociopolitiques d'émergence et des usages doivent être mises au jour.

3 L'ouvrage est constitué de six chapitres et de brèves introduction et conclusion générales. Le chapitre 1 offre une analyse des «étymologies» de la performance, le pluriel s'imposant du fait de la polysémie du mot, entre son sens anglo-saxon (accomplir) et son sens latin (réaliser un exploit). Florence Jany-Catrice y soutient que, désormais, la performance désigne "non seulement les résultats, mais aussi les processus d'optimisation dans l'accession à ces résultats" (pp. 22-23). Or, paradoxalement, la variété de sens «se double d'une restriction qui lui est de plus en plus imposée » par son inscription dans le "registre chiffré et calculatoire» (p. 23). Florence Jany-Catrice présente ensuite les raisons de la crise des concepts classiques de croissance et de productivité : conventions de mesure fondées sur la production de biens matériels, ils sont mis en défaut par la montée des services et des préoccupations sociales et environnementales. Enfin, elle rend compte de la substitution, dans les revues académiques d'économie, du mot " productivité " par celui de "performance ». Pour intéressant qu'il soit, la finalité du chapitre n'apparaît au bout du compte pas clairement: est-ce de montrer comment se résout, en pratique, la tension entre la mesure quantifiée, avec ce qu'elle a d'unidimensionnel, et la polysémie de la performance ? Ou est-ce, comme le contenu de la conclusion du chapitre l'indique, de montrer les raisons de la crise des concepts de croissance et de productivité ? Le lecteur ne parvient pas à s'y retrouver et peut être dubitatif quant à la portée de la découverte du concept juridique anglo-saxon de "performance ", qui renvoie à l'exécution d'un contrat.

4 Nettement plus convaincant et cadré, le chapitre 2 présente de manière dense et très argumentée les problèmes et enjeux de l'approche de la performance dans les économies de services. Outre le fameux «paradoxe de Solow » (qui indique l'absence de traces de l'informatisation dans les statistiques de la productivité) et les difficultés à élaborer une évaluation macroéconomique de la valeur ajoutée des services, il est question dans ce chapitre de la contre-performance des raisonnements en volume dans certaines activités de service : la recherche académique et les services d'aide à domicile. Florence Jany-Catrice conclut en pointant les limites de la substantification de la performance, via l'objectivation passant par la mise en place d'indicateurs.

Le chapitre 3 est consacré aux relations de travail. Florence Jany-Catrice montre que la recherche de la performance totale par la mise en œuvre de dispositifs d'incitation suppose à la fois une individualisation des relations de travail et des dispositifs de mesure ; dans le même temps, le lien entre performances individuelles et performances des organisations (en d'autres termes les performances collectives) devient problématique. En effet, «de même qu'une organisation ne peut être réduite à une collection d'individualités, la garantie de performance individuelle ne peut assurer de garantir la performance globale » (p. 50).

6 Passant des relations de travail à l'État, le chapitre 4 traite de la gestion publique, plus précisément du Nouveau Management public. Florence Jany-Catrice retrace l'entrée en scène des indicateurs de performance de l'État en les situant, à juste titre, dans le contexte de ses transformations, plus précisément la substitution d'un État 
«modernisé », prestataire de services, à l'État social de naguère. Plus original, le glissement de l'évaluation des politiques publiques vers la mesure de performance des services publics est un point fort de ce chapitre qui retient particulièrement l'attention.

$7 \quad$ La suite de l'ouvrage remonte en généralité. Le chapitre 5 synthétise les grands enjeux de la quantification; le chapitre 6 aborde avec une grande clarté les dimensions de démocratie dans la définition d'un régime de performance parmi d'autres. Rappelant que la quantification suppose des « investissements de forme » et un travail de « mise en équivalence ", l'auteure soutient dans le chapitre 5 que la mesure peut être mise en œuvre dans deux perspectives très différentes: celle de la « ratiocratie » qui fait de la mesure de la performance une "activité mécanique et aveugle»; celle, moins bien précisée par l'auteure, d'un "travail raisonné ", adossé à l'étude du réel, susceptible d'une appropriation par les acteurs (pp.121-122). Le chapitre 5 se conclut sur des réflexions concernant le rapport entre État, statistique et démocratie, thématique qui est au cœur du chapitre 6.

8 Le dernier chapitre est donc consacré aux conditions d'une production de la performance qui satisfasse une exigence démocratique, c'est-à-dire qui associe négociation et délibération. L'auteure centre son propos sur l'idée de "pluralité de régimes de performance ", envisagée sous deux angles : d'abord celui du processus de production du dispositif de performance (imposé ou négocié); ensuite celui du caractère uni ou multidimensionnel des critères de mesure et d'évaluation de la performance. Florence Jany-Catrice propose une typologie des régimes de performance sur la base de ces deux critères. Parmi les quatre types qu'elle dégage (autocratique, fordiste, managérial, délibératif), le dernier n'est pas de l'ordre des réalités : il « reste en partie à inventer » (p.154). Le point de vue de l'auteure est ici normatif: seuls des processus participatifs peuvent contribuer à l'élaboration de ce que doit être l'utilité sociale, parce qu'ils en assurent la légitimité. Mais les "grandeurs quantitatives » ne sauraient être exclusives d'autres formes de mesure, le qualitatif - assuré par des enquêtes sociologiques de trajectoires - devant prendre en compte " tout ce qui n'est pas mesurable arithmétiquement» et "peut légitimement être reconnu comme contribution sociale, comme une plus-value pour la société » (p. 156).

Florence Jany-Catrice offre dans cet ouvrage une excellente synthèse des questions et enjeux économiques, sociaux et politiques de la quantification et de la mesure. Le propos est pluridisciplinaire : l'auteure, professeure d'économie, emprunte beaucoup à la sociologie de la quantification, à la socioéconomie de l'économie des conventions, à la sociologie politique. On comprend aisément que le sujet justifie pleinement une logique d'objet, qui implique que les savoirs pertinents doivent être trouvés là où ils sont produits, c'est-à-dire dans plusieurs champs disciplinaires.

10 On peut regretter que Florence Jany-Catrice ne tire pas jusqu'au bout le fil de l'analyse des dispositifs de mesure comme base de coordination, comme elle l'annonce à plusieurs reprises ; or, l'idée que « la performance devient... un véritable processus de coordination des actions » (p. 78) est intéressante et originale. Elle supposerait que soit mise en place une étude de la manière dont les agents (dans leur diversité) se comportent au regard des dispositifs d'évaluation et de mesure qui constituent leur contexte d'action. C'est là une question sociologique majeure, pour laquelle on ne trouve pas dans l'ouvrage une contribution significative. On est également un peu sceptique sur la mise en équivalence analytique de la mesure de la productivité industrielle et, par exemple, la gestion publique basée sur des indicateurs de 
performance. Il est légitime de questionner la pertinence pour le secteur des services d'une mesure de la productivité conçue dans l'industrie. Mais c'est une autre question que Florence Jany-Catrice met en avant: la performance est devenue l'objet de la construction d'indicateurs qui posent, comme pour la statistique publique, le problème des conventions d'équivalence. Or, ces conventions comprennent une dimension technique, certes, mais aussi politique.

11 Si l'on devait définir l'unité de l'ouvrage, on ne pourrait manquer de la trouver dans la question politique et, partant, des conditions d'une élaboration démocratique et délibérative de la performance et des dispositifs de sa qualification. En cela, Florence Jany-Catrice place son ouvrage sur le registre de l'économie politique. Ce n'est pas la moindre de ses qualités.

\section{AUTEURS}

\section{THIERRY KIRAT}

Institut de recherche interdisciplinaire en sciences sociales (Irisso), université Paris-Dauphine 